\title{
Infective arthritis secondary to bladder outflow obstruction
}

\author{
S.S. Ubhi and T.J.C. Cooke \\ Department of Surgery, Salisbury General Infirmary, Salisbury, Wilts, UK
}

\begin{abstract}
Summary: We describe two cases of septic arthritis occurring in association with lower urinary tract infection in elderly men. In both cases the organism isolated from both the joint and the urine was Staphylococcus aureus. Further investigation of the urinary tract in both individuals identified bladder outflow obstruction secondary to benign prostatic hyperplasia predisposing them to infection.

The urinary tract should be suspected as a focus of infection in septic arthritis in elderly men and further investigation of the urinary system may disclose a surgically correctable lesion.
\end{abstract}

\section{Introduction}

It is unusual for septic arthritis to occur in previously normal joints; ${ }^{1}$ the organism, most commonly Staphylococcus aureus, usually gains access to the joint via the bloodstream. A search should be made for the primary focus of infection in such cases.

We describe two cases of infective arthritis due to Staphylococcus aureus in elderly men with previously healthy joints; we believe that in both cases the joint infection was secondary to bladder outflow obstruction due to benign prostatic enlargement complicated by Staphylococcus aureus lower urinary tract infection.

\section{Case reports}

Case 1

A 74 year old man presented with a 3-month history of an increasingly painful right ankle. He had suffered no trauma or injury to the joint.

On examination he was afebrile, the ankle was swollen and all movements were restricted by pain. An ankle X-ray showed no bony abnormality, the ESR was elevated at $121 \mathrm{~mm} / \mathrm{h}$ and the haemoglobin $9.7 \mathrm{~g} / \mathrm{dl}$. Aspiration of the joint yielded no fluid, and the symptoms persisted despite oral treatment with a non-steroidal anti-inflammatory drug.

He was therefore admitted for a synovial biopsy. On further questioning he admitted to increasing symptoms of bladder outflow obstruction over the

Correspondence: S.S. Ubhi, F.R.C.S., Department of Surgery, Leicester General Hospital, Gwendolen Road, Leicester LE5 4PW, UK.

Accepted: 12 June 1990 preceding 3 years. At the time of biopsy he was found to have an infective arthritis, the causative organism was identified as Staphylococcus aureus. The same organism was isolated from a mid-stream specimen of urine taken pre-operatively.

Further investigation of his urinary tract confirmed bladder outflow obstruction due to an enlarged prostate. A transurethral prostatectomys was performed for benign disease and he now remains well with no further urinary infection.

\section{Case 2}

A 73 year old non-insulin dependent diabetic man presented with a 3-day history of a painful swollen left knee and a 5-day history of burning dysuria with frequency of micturition. He was afebrile, the knee was swollen and all movements were restricted by pain.

He had suffered long standing symptoms of bladder outflow obstruction and many years previously had had transurethral removal of bladder calculi.

Pus was aspirated from the knee joint and a mid-stream specimen of urine was taken. Laboratory cultures identified $S$. aureus in both specimens.

Further investigation of his urinary system confirmed bladder outflow obstruction due to an enlarged prostate. A prostatectomy was performed for benign disease and he remains well with no further urinary infection.

\section{Discussion}

Staphylococcus aureus is an unusual infective micro-organism in the lower urinary tract, 
accounting for $1.5 \%$ of all nosocomial urinary tract infections, ${ }^{2}$ its presence suggests an underlying urinary tract abnormality. ${ }^{3}$

Predisposing factors to urinary infection in elderly men include prostatic enlargement, with incomplete emptying of the bladder after voiding, and the age-related diminution of antibacterial activity of prostatic secretions which reduce host defences against urinary infection. ${ }^{4}$

$S$. aureus bacteruria may be associated with instrumentation of the urinary tract and will develop into a secondary bacteraemia in $5.5 \%$ of the elderly male population. ${ }^{5,6}$ The classic symptoms of urinary infection may be absent and the diagnosis therefore delayed. ${ }^{7}$

Staphylococcal bacteraemia is well recognized following dental procedures, upper gastrointestinal endoscopy, urological procedures and parturition. ${ }^{8}$ The bacteria may localize in a number of sites around the body such as diseased heart valves.

The commonest pathogen in septic arthritis is $S$. aureus, the organism gaining access to the joint by haematogenous spread from a remote focus; less commonly the infection spreads from an adjacent focus, especially osteomyelitis, or by direct innoculation through a wound.

Septic arthritis usually occurs in joints that have had previous surgery or are affected by arthritic

\section{References}

1. Schmid, F.R. Bacterial arthritis. In: McCarty, D.J. (ed.) Arthritis and Allied Conditions. Lea \& Febiger, Philadelphia, 1979.

2. Jarvis, W.R., White, J.M., Munn, V.P. et al. Nosocomial Infections Surveillance 1983. $M M W R$ 1985, 33: 14SS.

3. Maskell, R. Urine microscopy and culture in selection of patients for urinary tract investigation. $B r J$ Urol 1989, 63: 7-10.

4. Lipsky, B.A. Urinary tract infections in men. Epidemiology, pathophysiology, diagnosis and treatment. Ann Intern Med 1989, 110: $138-150$.

5. Lee, B.K., Crossley, K. \& Gerding, D.N. The association between staphyloccal bacteremia and bacteruria. Am J Med 1978, 65: 303-306. disorders and it is unusual for it to occur in a previously healthy joint. ${ }^{1}$ Late infection of joint replacements, although rare, may be due to haematogenous spread of organisms from a remote site including the urinary system. ${ }^{9}$

Urinary infections are an important cause of sepsis in the elderly. In a survey of 100 cases of bacteraemia in the elderly, the urinary tract was the most frequent site of origin (56\%) and 12 episodes were associated with urinary catheters. ${ }^{10}$

Both of our patients had staphylococcal urinary tract infections. One of them was asymptomatic, and both presumably had an episode of bacteraemia which resulted in an infective arthritis in a previously normal joint.

We suggest that in patients, especially elderly males, presenting with septic arthritis the urinary tract should be considered as a possible source of the infection. Where this is shown to be the case appropriate urological investigations may uncover a surgically correctable lesion.

\section{Acknowledgements}

We wish to thank Messrs J.E. Carvell and G.F. Rushforth, consultant orthopaedic surgeons, for permission to report on their patients.
6. Demuth, P.J., Gerding, D.N. \& Crossley, K. Staphylococcal bacteruria. Arch Intern Med 1979, 139: 78-80.

7. Kunin, C.M. Detection, Prevention and Management of Urinary Tract Infections. Lea \& Febiger, Philadelphia, 1987.

8. Everett, E.D. \& Hirschmann, J.V. Transient bacteremia and endocarditis prophylaxis. A review. Medicine 1977, 56: 61-77.

9. D’Ambrosia, R.D., Shoji, H. \& Heater, R. Secondarily infected total joint replacements by hematogenous spread. $J$ Bone Joint Surg (Am) 1976, 58A: 450-453.

10. Setia, U., Serventi, I. \& Lorenz, P. Bacteremia in a long term care facility. Arch Intern Med 1984, 144: 1633-1635. 